\title{
Short Implants in Posterior Maxilla in Elderly Patients: A Case Series
}

\author{
David Buntoro Kamadjaja \\ Department of Oral and Maxillofacial Surgery, Faculty of Dental Medicine, Universitas Airlangga, Surabaya, Indonesia
}

\begin{abstract}
Placement of dental implants in reduced bone in the posterior maxilla requires maxillary sinus floor elevation. However, in elderly patients this is to be avoided. A case series on the successful placement of multiple short implants in posterior maxilla and splinted crown restorations in elderly patients was presented. Long term follow up revealed survival of the implants. Short implant is a suitable treatment option for elderly patients.
\end{abstract}

Key Words: reduced bone dimension, posterior maxilla, short implant, primary stability, implant diameter

\section{INTRODUCTION}

Paper presented at the Joint Scientific Meeting in Special Care Dentistry, July 5, 2019, Amerta Room, 4th Floor, main campus of Universitas Airlangga, Surabaya, Indonesia.

Corresponding author: David Buntoro Kamadjaja Department of Oral and Maxillofacial Surgery Faculty of Dental Medicine Universitas Airlangga

Jl. Mayjen. Prof. Dr. Moestopo No. 47 Surabaya 60132 - Indonesia Email: david-b-k@fkg.unair.ac.id
Dental implants have been an ideal treatment solution for patients who require permanent replacement of their missing teeth. Dental implants can be placed successfully in most cases, however, certain clinical situations, such as reduced bone dimension, may pose a significant challenge. This situation is frequently encountered in the posterior maxillary bone, where, following tooth extraction, sinus pneumatization often results in inadequate vertical bone height for implant placement. Sinus floor elevation using lateral window and bone grafting before implant placement has been the gold standard with highly predictable results. ${ }^{1}$ However, in elderly patients sinus floor elevation is to be avoided as much as possible due to systemic problems and associated delayed healing. In such situations, a less invasive surgery such as the use of short implants is more suitable.

An alternative method for the treatment of atrophic bone in posterior maxilla involves the use of short dental implants. Short implants are referred to as implants with the length of less than $10 \mathrm{~mm}$. The use of short implants has been controversial with regard the survival rates, especially as long as long-term survival of the dental implants is concerned. ${ }^{2}$

This case series aims to provide information on the successful treatment involving the use of short dental implants in three elderly patients. Few considerations and certain techniques in the implant placement and design of the final restoration were taken into account in achieving long term implant stability and survival.

\section{CASE REPORTS}

Case 1. A 65-year-old male patient came with edentulism at 16 following extraction two months earlier. Radiographically, vertical bone height at 16 region was not more than $5 \mathrm{~mm}$ (Figure 1A). Two-stage implant 
placement with the use of short implant $(5.2 \times 6.5 \mathrm{~mm}$, Axiom ${ }^{\circledR}$, Anthogyr, France) was placed in this type IV bone area. Crestal approach sinus floor elevation was performed gaining $2 \mathrm{~mm}$ apically (Figure 1B). Five months later, re-entry was done and healing abutment placed and maintained for one month. Final restoration was done with the insertion of standard abutment and porcelain fused metal (PFM) crown. Seven years follow-up revealed that the patient had been functioning with the implant-supported crown and clinically, the crown was stable. Radiograph taken demonstrated good implant osseointegration with normal bone level around the implant platform (Figure 1C).

Case 2. A 63-year-old male patient came with chief complaint of difficulty in chewing on his right teeth after extraction of his upper right molar. On examination, the 17 (retainer of bridge) was missing, 16 was pontic of bridge hanging onto the retainer at 15 . It was decided to remove the pontic and put two implants at 16 and 17 regions. Radiograph showed minimum amount of residual bone in vertical dimension (Figure 2A). Two short implants (Axiom ${ }^{\circledR}$, Anthogyr, France) of $6.5 \mathrm{~mm}$ and $8 \mathrm{~mm}$ were placed at type IV bone in the region of 17 and 16, respectively. At second stage 2 months later, re-entry was performed and healing screw placed followed by placement of standard abutments on which 2 units splinted PFM crowns was cemented (Figure 2B). At 6 year follow-up, there was no specific complaint, clinically the crowns were firm and problem-free. Radiograph showed good implant osseointegration and it was noted that vertical dimension of the alveolar bone in the respective sites were somewhat preserved (Figure 2C).

Case 3. A 72-year-old, came to have implant treatment to replace missing 14,15 and 16 following extraction two months earlier. Intra operatively, poor bone healing and severe bone defect at 15 region was found. It was decided then to place 2 implants at 14 and 16. In the former site, a regular implant (Axiom ${ }^{\circledR}$, Anthogyr, France) was placed while in the latter site, which was found to be of type IV bone, short $(5.2 \times 6.5 \mathrm{~mm})$ implant was chosen because of minimal bone height due to the large anthral floor (Figure $3 \mathrm{~A})$. At the second stage, standard abutments were inserted and three units porcelain fused to metal (PFM) bridge was cemented thereon (Figure 3B). Seven years later, the followup $x$-ray showed that both the implants survived and no signs of peri-implant bone loss were noted (Figure 3C).
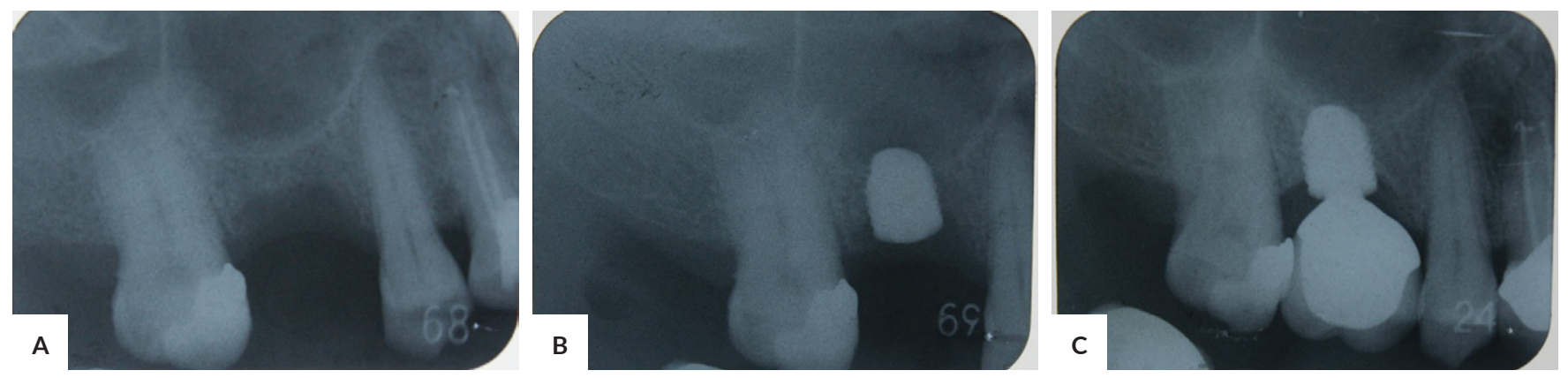

Figure 1. Single short implant in posterior maxilla. A 65-year-old male patient came with (A) edentulism at 16 site showing severe vertical bone resorption, (B) short $(6.5 \times 5.2 \mathrm{~mm})$ implant was placed involving the use of transalveolar sinus floor elevation, (C) implant stability and minimal bone remodelling around the implant were evident 7 years after implant placement.
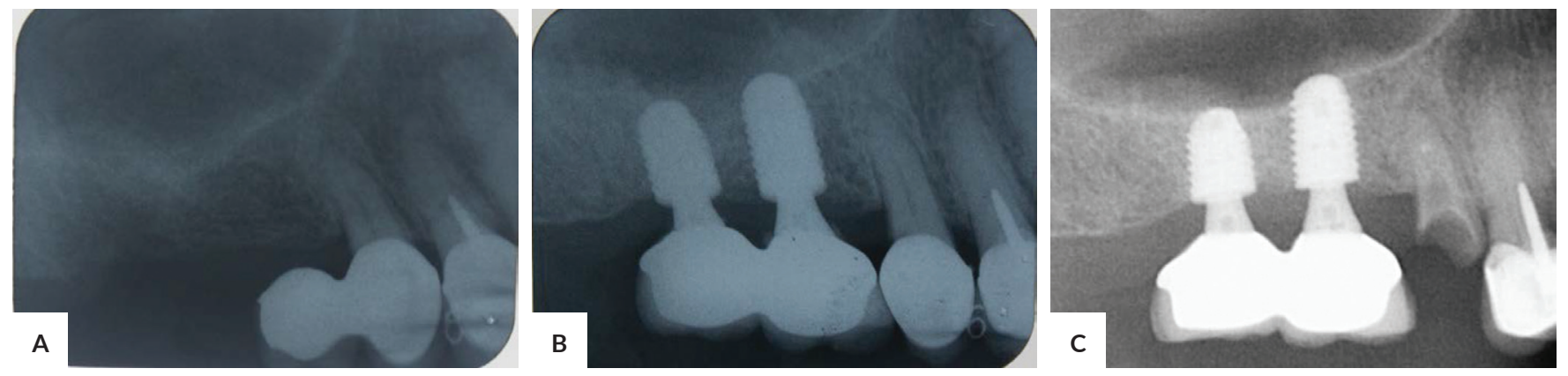

Figure 2. Double short implants in reduced bone dimension in posterior maxilla. A 63-year-old male patient required two implants in the right upper jaw. (A) In the radiograph minimal bone height was noted in the 16 and 17 sites, (B) two short implants, 6.5 and $8 \mathrm{~mm}$, respectively were placed and splinted one another with ceramic crowns, (C) at 6 years follow up, radiograph showed good implant osseointegration and clearly demonstrated preservation of the vertical dimension of alveolar bone in the respective sites. 

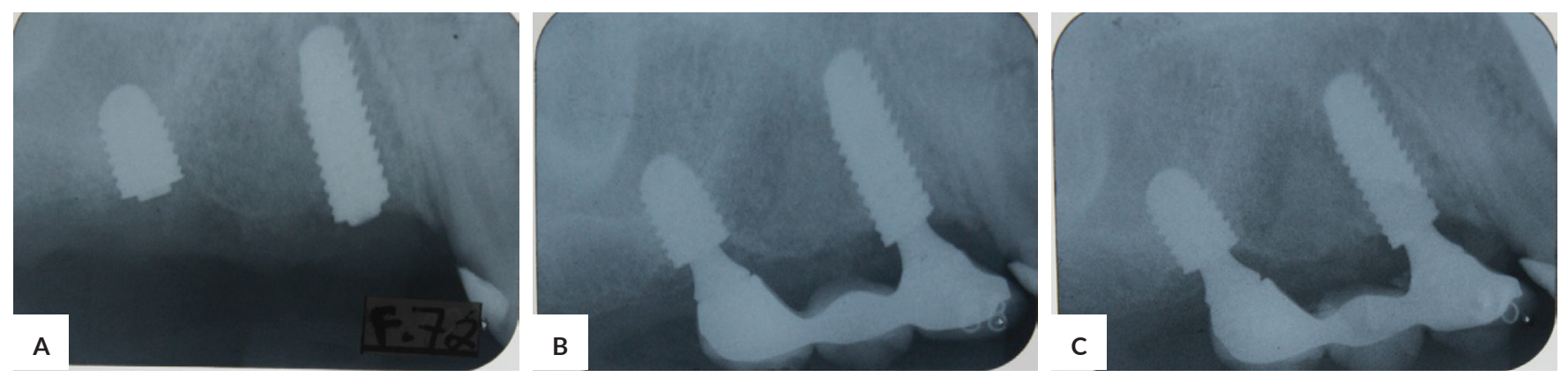

Figure 3. Short implants in combination with regular implant in atrophic posterior maxilla. A 72-year-old patient came with missing 14, 15 and 16 two months post extraction (A) Due to low vertical dimension of bone at 16 site, an implant of $6.5 \mathrm{~mm}$ length and $5.2 \mathrm{~mm}$ wide was placed in 16 region together with regular length implant in 14 region avoiding placement of implant at 15 site due to severe bone atrophy. (B) At second stage, final abutment and 3 units porcelain fused to metal bridge restoration was placed over the implants. (C) seven year later the implants were stable and no sign of peri-implant bone loss was noted.

\section{DISCUSSION}

There has long been a strong belief that long dental implant is important for the success of implant treatment: the longer the implants the higher the survival rates would be and hence a more favourable prognosis. However, in many situations such as in alveolar ridge deficiencies in posterior maxilla this is not always possible. Alveolar bone augmentation and sinus lift surgery involving the use of bone graft have been used with success. However, the procedures are time consuming and are associated with high cost and morbidity. ${ }^{2}$ This invasive surgery should not be considered as standard procedure for elderly patients where healing and the risks of complications are a concern.

Short dental implants have been proposed as an alternative treatment to simplify implant placement procedures in severely defective alveolar ridge, to avoid vital structures, minimize surgical trauma and the risk of complications associated with invasive surgical procedures. ${ }^{3}$ With this in mind, short dental implants would be more suitable in elderly patients. Short dental implants have often been associated with lower success rates compared with standard implants. However, such relationship has not been proven scientifically. Instead, many studies have shown that short dental implants have not demonstrated the increased risk of failure compared to longer implants. ${ }^{3}$

Many factors such as implant surface, implant primary stability, bone density and quantity, surgical preparation techniques used, management of the soft tissue and prosthodontic design ${ }^{4}$ have been related to implant survival rates. In such low bone density area as in these cases, biological and mechanical considerations should be taken into account. Biologically, a good primary implant stability is important, which can be achieved by under-prepared osteotomy site with the drilling stopped at diameter below the implant diameter. A two-stage implant placement and longer waiting time for second stage treatment are strongly recommended to allow for complete implant osseointegration. These measures have been implemented in the cases presented which may be attributed to the long-term survival rate.

Many studies have shown that implant survival rates are affected primarily by bone-implant contact area. ${ }^{5}$ The contact area concerned is determined by such factors as implant length, taper, diameter and surface texture ${ }^{2}$. It is noteworthy therefore that primary stability may not be difficult to achieve with short implants as long as the diameter is large enough to increase bone-implant contact. Furthermore, the osseointegration of bone-implant interface can be increased with the use of rough surface implant. In one study, it was indicated that the role of diameter of the implant on survival rate is more important than the length of the implant. ${ }^{6} \mathrm{~A}$ review study also showed that there is no statistically difference in the survival rate between standard and short implant in edentulous patients. These may be the explanation to the high survival rates in the cases presented above. All of the short implants used have wide platform and fixture diameter $(5.2$ $\mathrm{mm})$. Additionally, the implants used have rough surfaces which resulted in increased bone-implant contact area.

When using short dental implants one should be aware of some risk factors that may increase stress such as a higher crown-implant length ratio, high bone density in the region, and higher bite force. Some methods are, therefore, required to decrease stress by minimizing the size of the crown in the bucco-palatal dimension to decrease the lateral force on the restoration, avoiding cantilever as much as possible, and splinting multiple implants together with splinted crowns. ${ }^{4}$

In case 2 presented above, the two short implants were splinted together with splinted crowns. This may have contributed to the long-term survival of the implants. Furthermore, in cases 2 and 3 above, the crowns and bridges in the bucco-palatal dimension were minimized in order to lower the stress of the lateral forces impinging on the implant during mastication. 
The crown-implant ratio in short implants may have risks of biomechanical problems such as overloading or non-axial loading which can cause crestal bone loss. ${ }^{7}$ To minimize the risks of such complications occlusal table reduction and flattening of the occlusal cusps should be done. This is also consistent with all reported cases above as some modifications were made in the prosthesis to increase favourable load distribution.

The long-term survival of the implants in the second and third reported cases were likely related with the splinting implants together and splinting short implants with the longer one. Studies showed that splinting short implants could increase the survival rate. ${ }^{5}$ Another study revealed that stress levels in bones surrounding splinted implants were significantly lower than those surrounding uncoupled implants. ${ }^{6}$

Bone quality and site of implant placement may also contribute to the treatment outcome. Some studies showed the short implant placed in the maxilla has a lower survival rate than those placed in the mandible. This could be secondary to bone density differences between the two jaws. This was also attributed to stress concentration reduction around the implant and improvement in the mechanical properties of the implant-bone interface, which consequently facilitated primary stability and early osseointegration, compensating for the reduction in the implant length.

\section{CONCLUSION}

The case reports showed long-term success in the implant treatments with the use of short implants in elderly patients. Dental implant treatments with short implant is suitable for elderly patients as it is considered minimally invasive surgery which can reduce the risks of surgical complications and compromised post-operative healing. To achieve long term short implant survival many factors such as good surgical technique, primary implant stability and prosthetic modifications to allow for stress reduction during mastication, should be considered.

\section{Statement of authorship}

The author has approved the final version submitted.

\section{Authors disclosure}

The author declared no conflict of interest.

\section{Funding source}

None.

\section{REFERENCES}

1. Papaspyridakos P, De Souza A, Vazouras K, Gholami H, Pagni S, Weber HP. Survival rates of short dental implants $(\leq 6 \mathrm{~mm})$ compared with implants longer than $6 \mathrm{~mm}$ in posterior jaw areas: A meta-analysis. Clin Oral Implants Res. 2018 Oct; 29 Suppl 16:820. doi: 10.1111/clr.13289

2. Esfahrood ZR, Ahmadi L, Karami E, Asghari S. Short dental implants in the posterior maxilla: a review of the literature. J Korean Assoc Oral Maxillofac Surg. 2017 Apr; 43(2): 70-6.

3. Mokcheh A, Jegham H, Turki S. Short implants as an alternative to sinus lift for the rehabilitation of posterior maxillary atrophies : Systematic review and meta-analysis. J Stomatol Oral Maxillofac Surg. 2019 Feb; 120(1):28-37.

4. Srinivasan M, Vazquez L, Rieder P, Moraguez O, Bernard JP, Belser UC. Survival rates of short $(6 \mathrm{~mm})$ micro-rough surface implants: a review of literature and meta-analysis. Clin Oral Implants Res. 2014 May;25(5):539-45. doi: 10.1111/clr.12125.

5. Thoma DS, Cha JK, Jung UW. Treatment concepts for the posterior maxilla and mandible: short implants versus long implants in augmented bone. J Periodontal Implant Sci. 2017 Feb;47(1):2-12. doi: 10.5051/jpis.2017.47.1.2.

6. Uehara PN, Matsubara VH, Igai F, Sesma N, Mukai MK, Araujo MG. Short Dental Implants $(\leq 7 \mathrm{~mm})$ Versus Longer Implants in Augmented Bone Area: A Meta-Analysis of Randomized Controlled Trials. Open Dent J. 2018 Apr; 12: 354-65. doi: 10.2174/1874210601812010354

7. Hasanoglu Erbasar GN, Hocaoğlu TP, Erbasar RC. Risk factors associated with short dental implant success: a long-term retrospective evaluation of patients followed up for up to 9 years. Braz Oral Res. 2019;33:e030. doi: 10.1590/1807-3107bor-2019.vol33.0030. 